\title{
Impact of COVID-19 on routine primary healthcare services in South Africa
}

\author{
Y Pillay, ${ }^{1} \mathrm{PhD}$; S Pienaar, ${ }^{1} \mathrm{PhD}$; P Barron, ${ }^{2} \mathrm{FFCH}(\mathrm{SA}) ; \mathrm{T}$ Zondi, ${ }^{3} \mathrm{MPH}$ \\ ${ }^{1}$ Clinton Health Access Initiative, Pretoria, South Africa \\ ${ }^{2}$ School of Public Health, Faculty of Health Sciences, University of the Witwatersrand, Johannesburg, South Africa \\ ${ }^{3}$ National Department of Health, Pretoria, South Africa
}

Corresponding author: Y Pillay (ygpillay@gmail.com)

Background. The COVID-19 pandemic and responses by governments, including lockdowns, have had various consequences for lives and livelihoods. South Africa (SA) was one of the countries that implemented severely restrictive lockdowns to reduce transmission and limit the number of patients requiring hospitalisation. These interventions have had mixed consequences for routine health services.

Objectives. To assess the impact of COVID-19 and restrictions imposed to limit viral transmission on routine health services in SA.

Methods. Data routinely collected via the District Health Information System in 2019 and 2020 were analysed to assess the impact of the COVID-19 pandemic.

Results. Access to public health services between March 2020 and December 2020 was limited in all provinces. However, this was not linear, i.e. not all services in all provinces were similarly affected. Services most severely affected were antenatal visits before 20 weeks, access to contraceptives, and HIV and TB testing. The impact on outcomes was also noticeable, with a measurable effect on maternal and neonatal mortality.

Conclusions. The responses to the COVID-19 pandemic, including different levels of lockdowns, the limitation of health services, lack of staff as a result of COVID-19 infection, and fear and stigma, resulted in a reduction in access to routine health services. However, the picture varies by type of service, province and district, with some faring worse than others. It is important to ensure that routine services are not significantly affected during future COVID-19 waves. This will require careful planning on the part of service providers and optimal communication with patients and communities.

S Afr Med J 2021;111(8):714-719. https://doi.org/10.7196/SAMJ.2021.v111i8.15786

This article looks at the impact of COVID-19 on several primarylevel healthcare services in the public sector in South Africa (SA) by comparing routine data from March 2020 to December 2020 with similar routine data from 2019.

The first patient laboratory diagnosed with SARS-CoV-2 in SA, on 5 March 2020, was a person who had returned from Europe. ${ }^{[1]}$ As the number of positive cases increased, President Ramaphosa, on advice from a range of technical experts, announced a 21-day nationwide lockdown with effect from midnight on Thursday 26 March 2020. Subsequently, the Cabinet, advised by the National Coronavirus Command Council, implemented several lockdowns with varying levels of restrictions to contain the spread of the virus. Fig. 1 shows the various lockdowns and changes in the number of COVID-19 cases. These lockdowns placed a series of restrictions on movement during the remainder of 2020 and into 2021, with significant consequences for health services.

At the global level, there have been many reports on the effects that lockdowns, and other restrictions resulting from COVID-19, have had on health services. In their World AIDS Day report in November 2020, the Joint United Nations Programme on HIV and AIDS (UNAIDS) ${ }^{[2]}$ reported that 'The COVID-19 pandemic is having far-reaching effects on health systems and other public services. In many countries, HIV services have been disrupted, and supply chains for key commodities have been stretched. Around the world fewer people are being diagnosed with HIV and fewer people living with HIV are starting HIV treatment.' Similarly, tuberculosis (TB) testing and TB notifications have been significantly affected by COVID-19. Data collated by the World Health Organization (WHO ${ }^{[3]}$ showed sharp drops in TB notifications in high-burden countries in 2020. In SA between February and May 2020, the National Institute for Communicable Diseases ${ }^{[4]}$ reported that during this period GeneXpert testing declined by $48 \%$ and positive specimens declined by $33 \%$.

In respect of reproductive health services, a 2020 survey of women of reproductive age in the USA ${ }^{[5]}$ found that one in three (33\%) reported that they had had to delay or cancel visiting a healthcare provider for sexual and reproductive healthcare because of the pandemic. Access to in-person healthcare had been severely limited, and people may have avoided seeking available services because of fear of contracting and spreading COVID-19. ${ }^{[5]}$ In SA, a review of data for the first half of $2020^{[6]}$ found that during the first wave of COVID-19, which largely coincided with lockdown levels 5 and 4 (the most stringent restrictions), there was a $30 \%$ increase in institutional maternal mortality. Although antenatal care attendance and in-facility deliveries were unchanged from 2019, use of contraceptive services and termination of pregnancy declined sharply. A review of maternal and child health services in Nigeria, Bangladesh and $\mathrm{SA}^{[7]}$ found that there was a reduction in utilisation of basic essential services such as antenatal care, family planning and immunisation due to the implementation of lockdown, which triggered fear of contracting COVID-19 and deterred people from accessing basic care, and a shift of focus towards the pandemic, to 


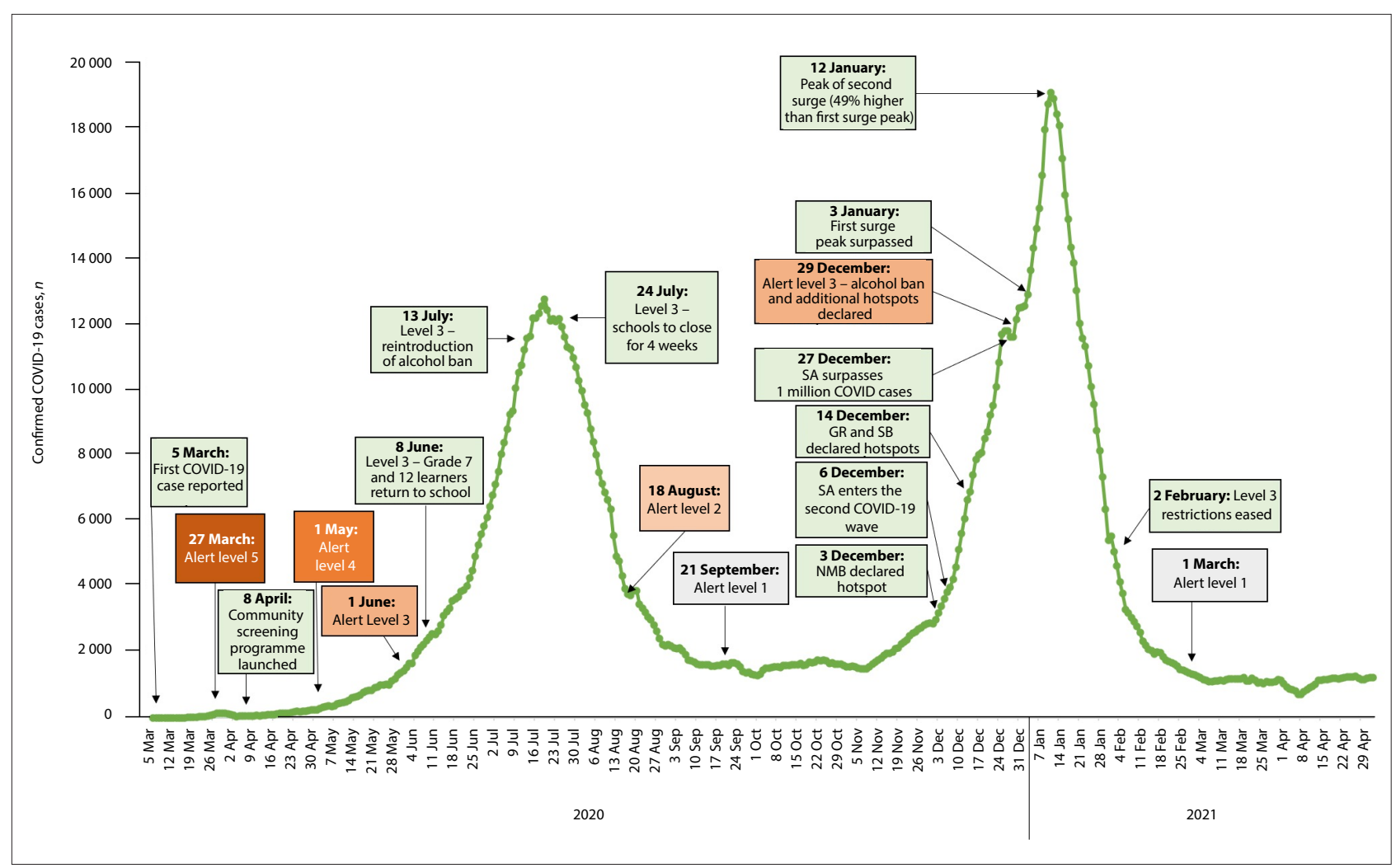

Fig. 1. Number of confirmed COVID cases in SA and key milestones from March 2020 through to April 2021. (SA = South Africa; NMB = Nelson Mandela Bay; $G R=$ Garden Route; $S B=$ Sarah Baartman.)

the detriment of other health services and causing resource constraints. Taken together, these factors resulted in compromised provision of basic healthcare.

A review of health systems factors suggests that many countries in Africa will see a significant drop in their immunisation $\operatorname{rates}^{[8]}$ as a result of lockdown-related factors such as access to health facilities, limited access to transportation and disruptions to the supply chain, as well as historical socioeconomic factors such as poverty.

In exploring the impact of COVID-19 on the number of laboratory specimens sent to the Tygerberg public sector laboratories in Western Cape Province, SA, it was found that there was a significant decline in the number of tests for non-communicable diseases between March and June 2020 compared with the same period in 2019. ${ }^{[9]}$

The above brief review of some of the literature reporting on the impact of COVID19 on routine primary-level healthcare services is limited to a short period (mainly March to June 2020) and specific facilities or geographical areas. In this article we analyse routine health services data for the whole of SA over a 10-month period from March 2020 to December 2020 and compare these with similar data for a corresponding period during 2019.

\section{Methods and data source} In $\mathrm{SA}$, routine data from the public sector (which caters for $\sim 85 \%$ of the total population) are available from the District Health Information System (DHIS). We analysed routine data for selected indicators for the period March 2020 - December 2020 and compared these with similar data for the same period in 2019 (pre-COVID-19). Data were analysed nationally (for SA) as well as subnationally (for the nine provinces). The data for GeneXpert tests conducted were obtained from the National Health Laboratory Service (NHLS), which serves the public health sector in SA.

Several indicators were chosen as proxy indicators for overall primary healthcare (PHC). These indicators represent the priority health programmes in SA. The indicators, with the service measured in brackets, were:

- total number of PHC visits (overall access to care)

- access to contraceptives (reproductive health)

- access to antenatal care (maternity care)

- number of babies delivered in public health facilities (maternity care)

- institutional maternal and neonatal mortality (maternity care)

- immunisation coverage (child health)

- number of HIV tests (HIV services)
- number of TB tests and people diagnosed with TB (TB services).

\section{Results}

\section{Number of PHC attendances}

This indicator represents the total number of patient visits at facilities during March 2020 and December 2020 compared with the same period in 2019. The data show a significant decline for every province during 2020. As shown in Fig. 2, the national headcount fell from 99.6 million visits in 2019 to 81.2 million in 2020, with all provinces experiencing a decline, the largest in Western Cape (31.1\%) and the smallest in Free State (8.7\%).

\section{Access to contraceptives and family planning}

Fig. 3 reflects the percentage difference in contraceptives prescribed for the March December periods in 2019 and 2020. The largest decline was in Mpumalanga (21.1\%) and the smallest in Free State (1.6\%). The largest declines were in April and May 2020 relative to the same period in 2019. This was the period during which lockdown levels 5 and 4 were in force. There was a further dip in provision of contraception in July 2020 compared with July 2019. Stockouts of certain products during 2020 also contributed to the decline in provision 


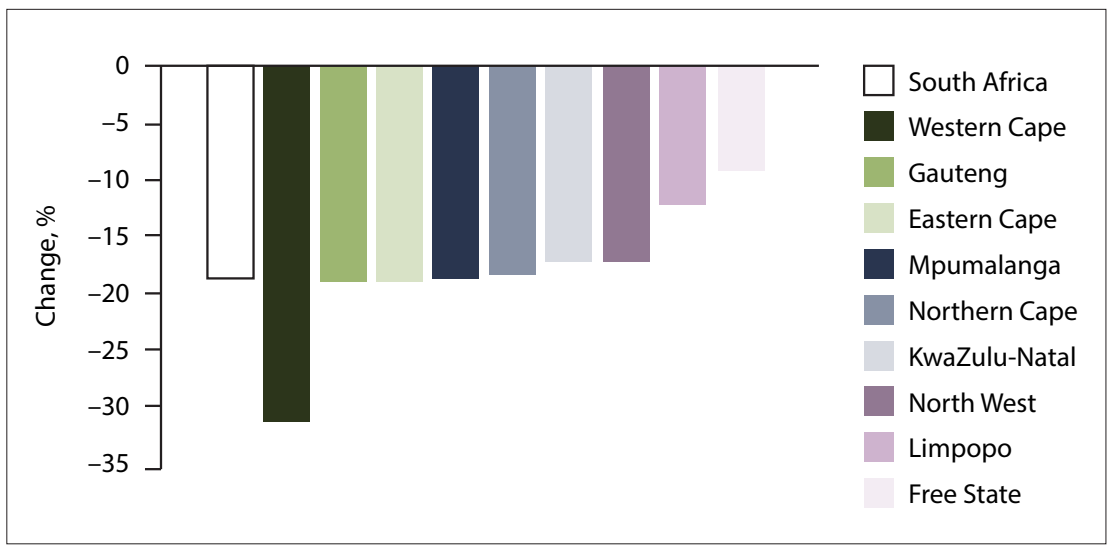

Fig. 2. Changes in numbers of patient visits between March and December 2019 and 2020.

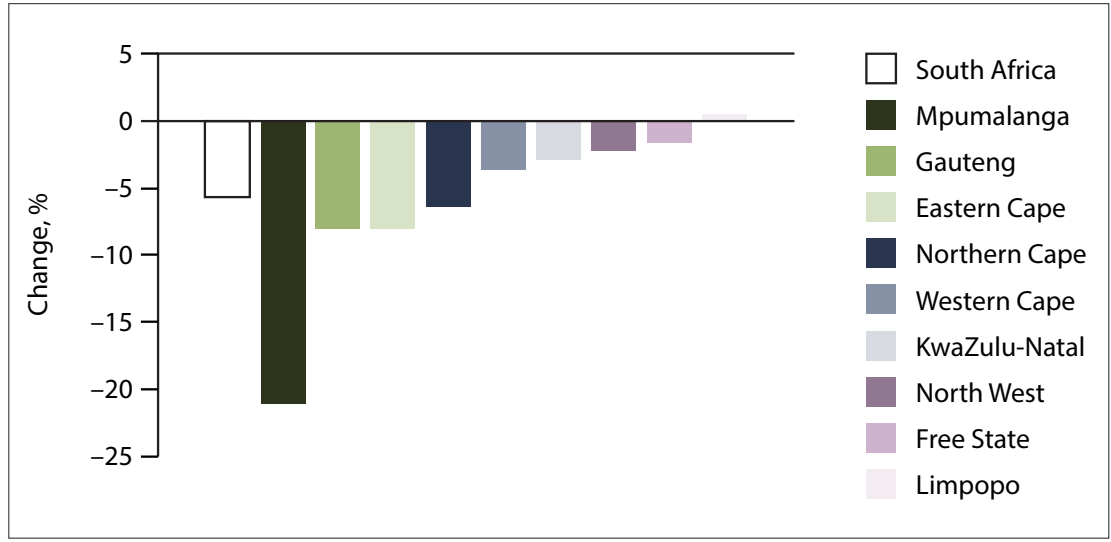

Fig. 3. Changes in contraceptive methods prescribed between March and December 2019 and 2020.

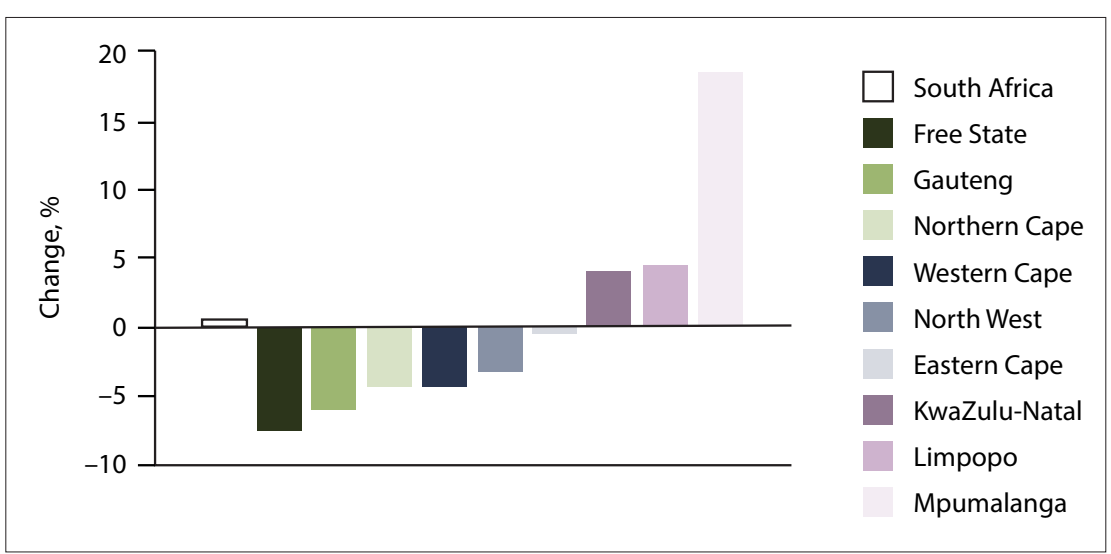

Fig. 4. Changes in first antenatal care visits before 20 weeks between March and December 2019 and 2020 .

of contraceptives. For example, the volume of medroxyprogesterone injections declined sharply in August 2020 compared with the same period in 2019. Healthcare workers substituted injectable with oral contraceptives during this period to replace those contraceptives that were out of stock.

Antenatal care visits before 20 weeks Access to antenatal care throughout pregnancy, but especially the first booking visit before 20 weeks, is important for good pregnancy outcomes. Fig. 4 reflects changes in the number of antenatal visits within the first 20 weeks of conception recorded during 2019 and 2020. Nationally, the number of first antenatal visits before 20 weeks was similar between 2019 and 2020, with some provinces having increased numbers while others had decreased numbers.

\section{Deliveries in public health facilities}

Fig. 5 shows the number of births during March - December for 2019 and 2020, with a $3.7 \%$ increase in deliveries in 2020 . Two provinces showed a decline, KwaZulu-Natal $(1.6 \%)$ and Free State $(0.8 \%)$, while the rest had increases of between $2 \%$ and $10 \%$, with the largest in Mpumalanga (10.4\%), Limpopo (8.4\%) and Gauteng (5.8\%).

\section{Institutional maternal mortality}

Nationally, 967 maternal deaths were reported during the period March - December 2020, compared with 788 during the same period in 2019. This is an increase of 179 deaths $(22.7 \%)$. As shown in Fig. 6, all provinces, with the exception of Northern Cape, which had small numbers and a 38.5\% decline, recorded increases in maternal mortality. The largest proportional increases were in Western Cape (82.1\%), KwaZuluNatal (44.2\%) and Mpumalanga (36.7). The institutional maternal mortality ratio increased from 90.5/100 000 live births for the period March - December 2019 to $106.8 / 100000$ for the same period in 2020 .

\section{Neonatal deaths in facility}

The neonatal period covers the first 28 days of life. Nationally, there was an increase of $487(4.8 \%)$ in institutional neonatal mortality between March and December 2019 and 2020. The institutional neonatal mortality rate increased from 12.0/1 000 live births in 2019 to $12.2 / 1000$ in 2020. Fig. 7 shows that most provinces had increases, with the greatest proportional changes in North West (25.1\%), Mpumalanga (10.2\%), Gauteng (9.5\%) and Western Cape (8.3\%).

\section{Immunisation coverage}

Fig. 8 shows that the number of children fully immunised at 1 year of age declined by $4.3 \%$ between 2019 and 2020. The largest decreases occurred during April, July and August 2020 compared with 2019. These months also coincided with periods of lockdown. Most provinces had declines, with the largest in Northern Cape (11.8\%), Eastern Cape (9.9\%) and Mpumalanga (8\%). North West was the exception, with an increase of $7.1 \%$.

\section{HIV testing}

Nationally, 3.44 million fewer HIV tests were done in March to December 2020 compared with 2019 (a 22.3\% decline). Apart from North West, which recorded a $1.7 \%$ increase in the number of HIV tests conducted, all provinces showed significant declines. Fig. 9 reflects the changes in tests done by province, with the largest declines in Western Cape (36.1\%), Gauteng (31.4\%) and Northern Cape (29.2\%). Fig. 10 shows the number of tests done each month during 2019 and 


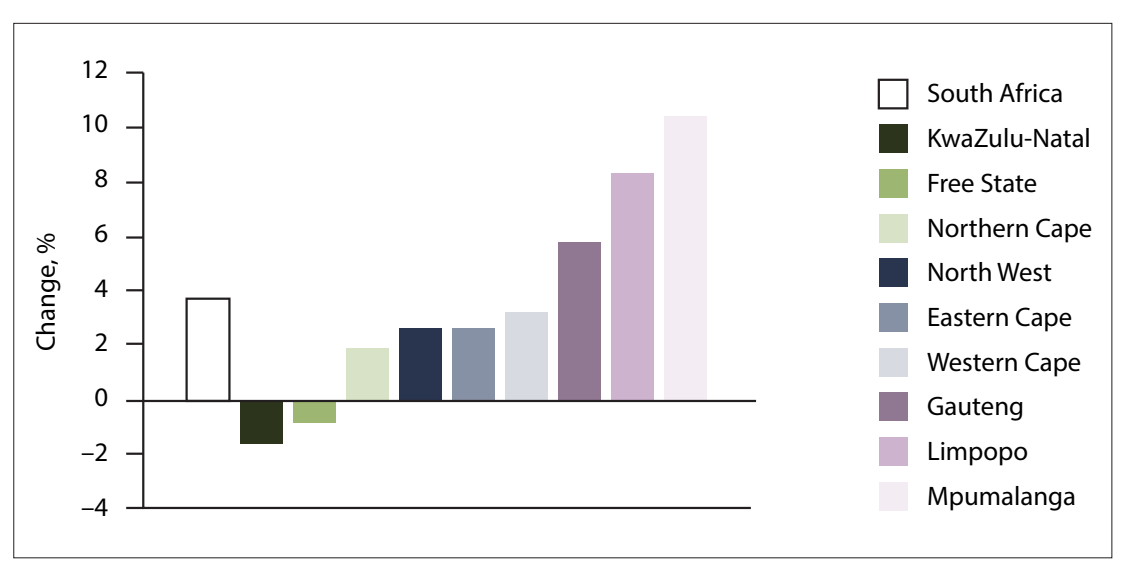

Fig. 5. Changes in number of births in public health facilities between March and December 2019 and 2020.

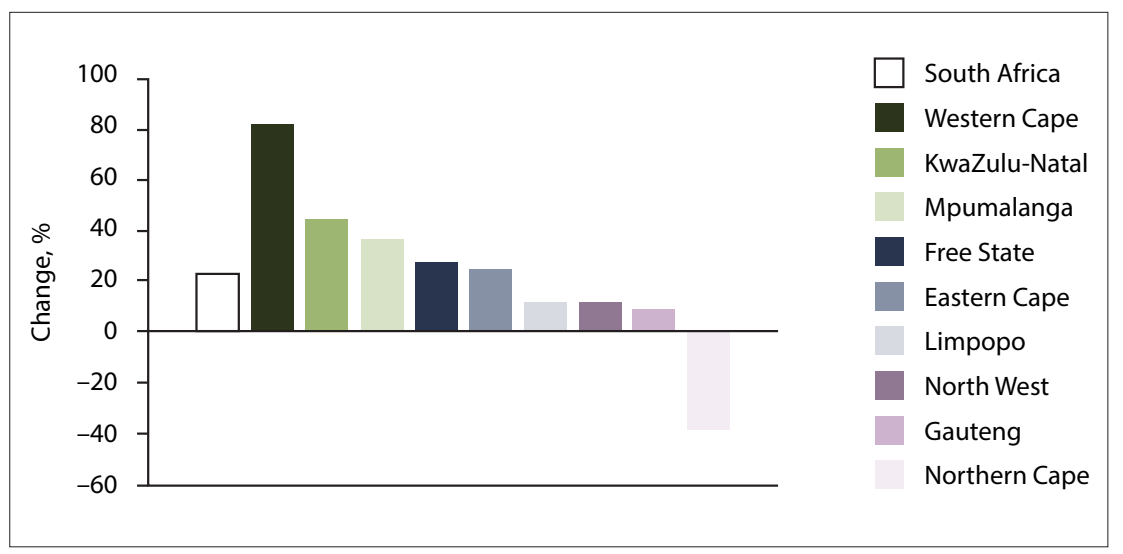

Fig. 6. Changes in maternal deaths in public health facilities between March and December 2019 and 2020 .

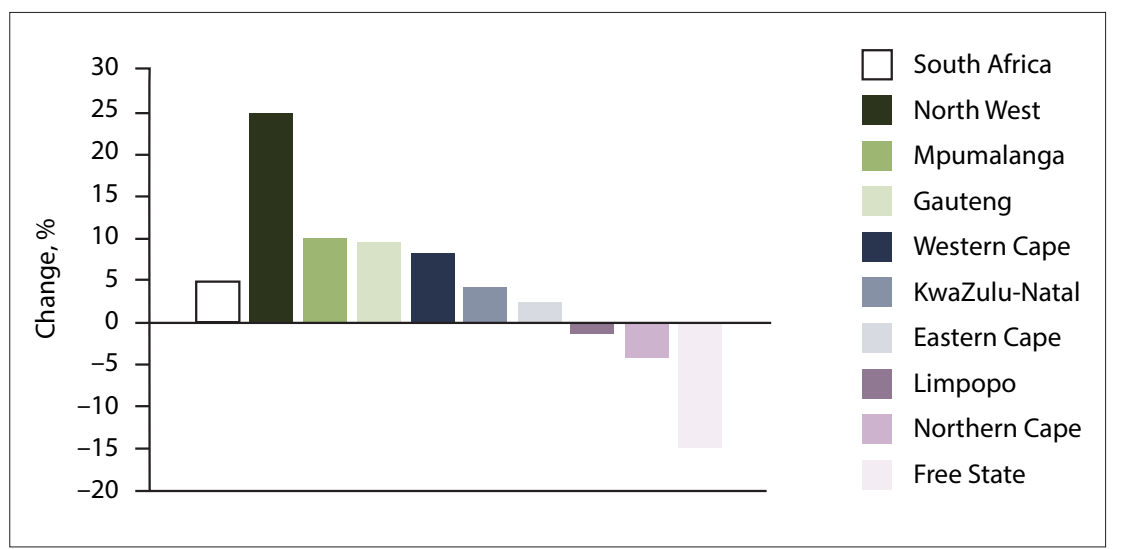

Fig. 7. Changes in institutional neonatal deaths in public health facilities between March and December 2019 and 2020.

2020. Each month in 2020 was lower than the corresponding month in 2019, with the period between April and July 2020, which corresponded to lockdown levels 5 to 3 , showing the largest decline in HIV tests done.

\section{Number of patients screened and tested for TB}

According to the routine statistics of the DHIS, the number of screenings for $\mathrm{TB}$ to February 2021. There was a precipitous drop in the number of tests done in April 2020 (coinciding with the start of level 5 lockdown), and by February 2021 the number of tests was still lower than the expected number.

\section{Discussion}

The routine data on selected primary care indicators in the public health system in SA over the period from the first COVID-19 case in March 2020 through to December 2020 give a clear picture of the negative indirect consequences in terms of use and quality of care. Almost across the board there was a decrease in use, with far fewer people visiting health facilities, using head counts (number of patient visits) as a proxy.

The likely reasons for a decline in headcounts, which was expected, include severe lockdowns, fear of contracting COVID-19 either on the way to or in health facilities, fear of transmitting the disease to families, and limited availability of transport. In addition, some facilities were closed during level 5 lockdown or only open for emergencies. There was also rationing and limited services because of staff shortages (staff being ill or in isolation, or redeployed to provide COVID-19-related services). These reasons for lower headcounts were also found in countries such as Bangladesh, Kenya, Nigeria and Pakistan. ${ }^{[11]}$

The decline in the number of contraceptives prescribed in most provinces is of major concern. It is likely that unplanned and unwanted pregnancies will increase, with negative consequences for both mothers and babies. The United Nations Population Fund (UNFPA) estimates that 12 million women globally were unable to access contraceptive services because of disrupted access, resulting in 1.4 million unintended pregnancies across 115 low- and middle-income countries. ${ }^{[12]}$

Although there was little change in the number of women booking for antenatal care during the first 20 weeks of pregnancy, there have been many reports of the negative impact of COVID-19 on the mental health of pregnant women. A review found that when infected with COVID-19, pregnant women had more severe symptoms. ${ }^{[13]}$ In addition, pregnant women experienced a range of mental health conditions, including anxiety and depression. ${ }^{[13]}$ The need for access to regular antenatal care may therefore increase during the pandemic, which implies that every effort should be made to ensure access to health facilities for pregnant women. 
Although mental health services are not discussed in this article because of the lack of robust, reliable data, there have been many reports of the adverse impact of COVID-19

The increases in maternal and neonatal mortality in many provinces reflect the direct and indirect impact of COVID-19. The direct impact on pregnant women relates to those who died from COVID-associated causes, while the indirect impact may relate to decreased access to and quality of care. The data used in this analysis do not permit a more granular analysis of the proportion of deaths related directly to COVID-19 disease and those related to inadequate access to and quality of care. In addition, the pressure on

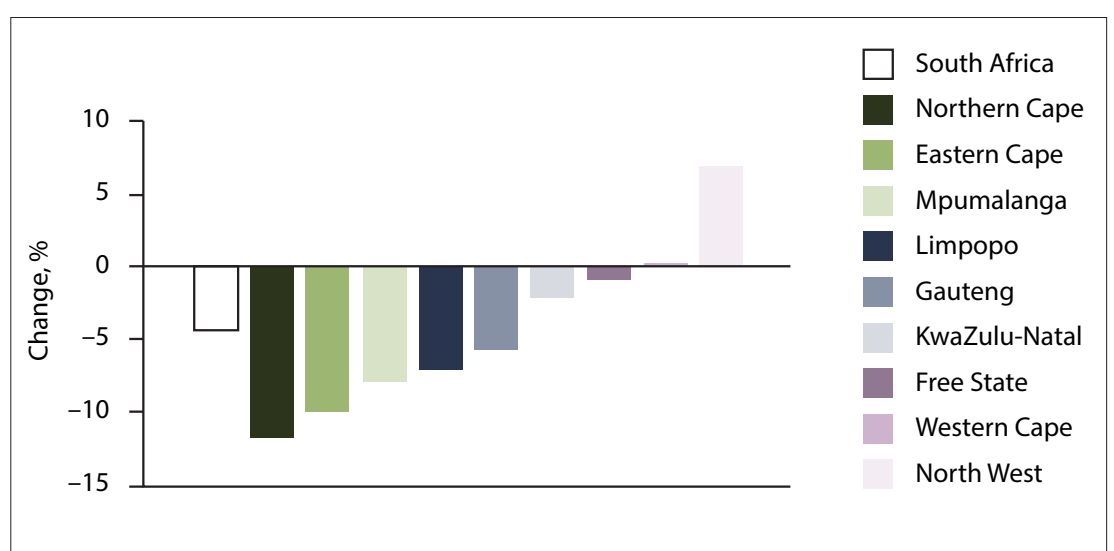

Fig. 8. Changes in children under 1 year fully immunised in public health facilities between March and December 2019 and 2020.

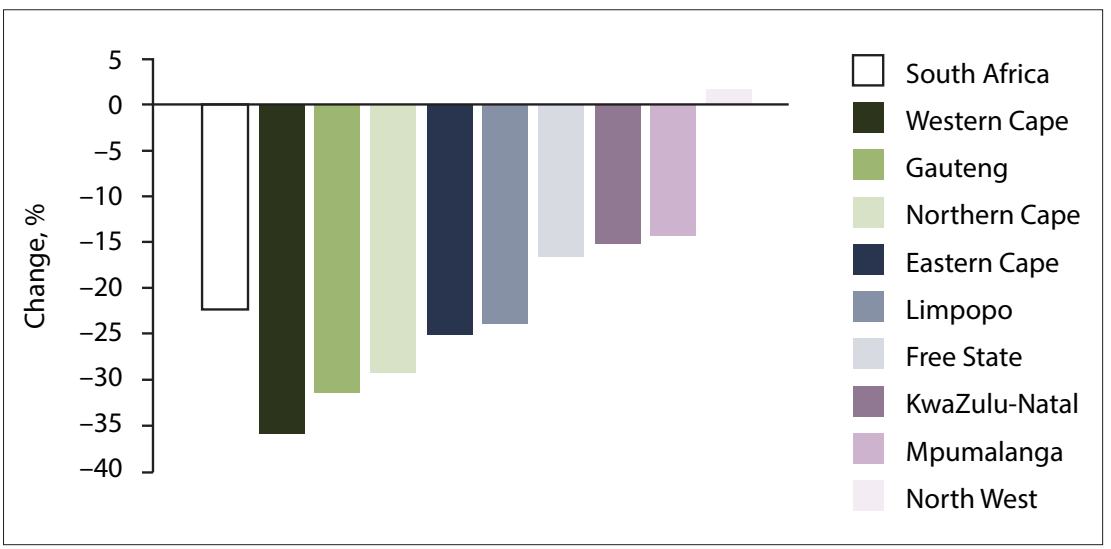

Fig. 9. Changes in HIV tests done in public health facilities between March and December 2019 and 2020 .

health facilities to treat COVID patients may have resulted in inadequate care provided to pregnant women and neonates, especially in rural and historically under-served areas, where quality was suboptimal prior to COVID-19.

Also of concern is the decline in the number of children who were fully immunised in 2020 compared with 2019. Seven of the nine provinces showed a decline, with only North West showing an increase in children aged under 1 year fully immunised. The impact of reduced access to services and reduced immunisation coverage has also been seen in other countries. ${ }^{[15]}$ To prevent an increase in vaccine-preventable diseases such as measles, provinces should

\section{South Africa}

Eastern Cape

Gauteng

KwaZulu-Natal

Free State

Western Cape

North West on mental health. ${ }^{[14]}$

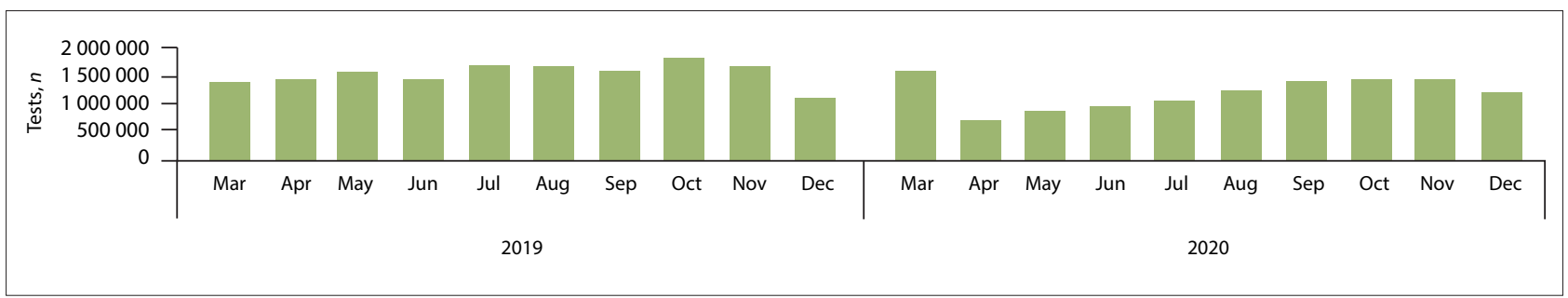

Fig. 10. HIV tests done by month in public health facilities between March and December 2019 and 2020 actively find under-immunised children and vaccinate them.

With nearly eight million people in SA infected with HIV, the largest number globally, testing and initiating people infected with HIV on treatment are particularly important to reduce transmission. ${ }^{[16]}$ The $22.3 \%$ decline in the number of people tested for HIV in 2020 means that fewer people living with HIV were initiated on treatment, resulting in SA not achieving the desired targets towards ending the AIDS epidemic. The decline in testing is similar for TB, with a $26 \%$ decrease in GeneXpert tests done in 2020. The major problem of eliminating $\mathrm{TB}$ in SA is finding the 'missing patients', especially those who are infected but not diagnosed. The decrease in testing numbers implies that the number of 'missing patients' will increase and add to the estimated 150000 people in SA with TB who are not on treatment. ${ }^{[17]}$

Findings similar to those reported in this article were also described in a review published by the Global Fund. ${ }^{[18]}$ In a study of 502 health facilities from countries in Africa and Asia, the Fund found that antenatal first visits declined by $43 \%$ in 2020 compared with 2019 , under- 5 child consultations by $74 \%$, HIV testing by $41 \%$ and referral for TB services by $59 \%$. Major reasons for these declines included fear, mistrust, lack of transport and severe lockdowns.

The WHO's pulse survey on the continuity of services during the COVID-19 pandemic of key stakeholders in 100 countries also found disruptions in services. ${ }^{[19]}$ Stakeholders listed seven approaches to mitigate the impact of COVID-19 on routine services: triaging to identify priorities; use of telemedicine as a substitute for in-person consultation; task shifting; supplying medicines through non-medical facilities; provision of information to communities on possible service changes; redirecting patients to health facilities that are open; and removal of user fees. Some of these strategies have been used in SA, including expansion of the centralised chronic disease dispensing and distribution system and the use of telephone consultation. 


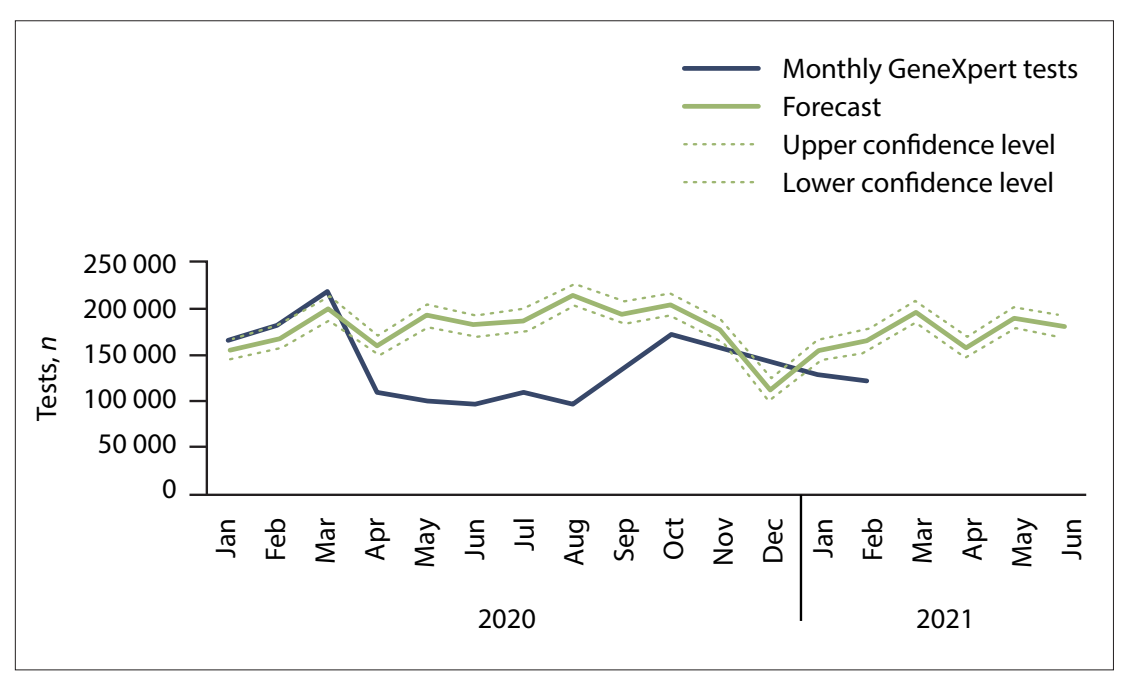

Fig. 11. Number of GeneXpert tests done for tuberculosis between January 2020 and February 2021 compared with the expected number (source: Moultrie et al. ${ }^{[10]}$ National Health Laboratory Service).

\section{Study limitations}

The major limitations of this article are due to reliance on a single data source, the DHIS, the quality of the DHIS data, and the use of a single year (2019) as a baseline comparator. In addition, the data are purely descriptive and no specific attributions are possible. Finally, data on non-communicable diseases were omitted owing to the poor and unreliable quality of the routinely collected data. ${ }^{[2]}$

\section{Conclusions}

While the impact of the COVID-19 pandemic on primary-level care has varied according to type of service delivered and geographical area (provinces), in most service areas there have been significant negative impacts on routine services. As SA plans for the next wave(s) and rolls out its vaccine plan, it is critical that routine services are protected. Failure to protect routine services such as general access to primary-level care including contraceptives, immunisation, and testing for TB and HIV will increase the burden of disease and associated morbidity and mortality.

\section{Declaration. None,}

Acknowledgements. None.
Author contributions. YP conceptualised and drafted the manuscript. SP produced the figures, and $\mathrm{PB}$ and $\mathrm{TZ}$ revised and edited the

Funding. None.

Conflicts of interest. None.

1. National Institute for Communicable Diseases. Impact of COVID-19 intervention on TB testing in South Africa. 10 May 2020. https://tbsouthafrica.org.za/sites/default/files/ news/20200510\%20Impact-of-Covid-19-interventions-on-TBtesting-in-South-Africa-10-May-2020.pdf (accessed 3 April 2021)

2. Joint United Nations Programme on HIV and AIDS (UNAIDS). Prevailing against pandemics by putting people at the centre. Geneva: UNAIDS, 2020. https://www.unaids.org/ sites/default/files/media_asset/prevailing-against-pandemics en.pdf (accessed 3 April 2021).

3. World Health Organization. Global Tuberculosis Report 2020. Geneva: WHO, 2020. https://www.who.int/publications/

4. National Institute for Communicable Diseases. Impact of COVID-19 intervention on TB testing in South Africa. 10 May 2020. https://tbsouthafrica.org.za/sites/default/files/ 10 May 2020. https://tbsouthafrica.org.za/sites/default/files/
news/20200510\%20Impact-of-Covid-19-interventions-onnews/20200510\%20Impact-of-Covid-19-interventions-on-
TB-testing-in-South-Africa-10-May-2020.pdf (accessed 3 April TB-testin.

2021).
5. Lindberg LD, van der Vusse A, Muelle J, Kirstein M. Early impacts of the COVID-19 pandemic: Findings from the 2020 Gutmacher survey on reproductive health experiences. New York: Guttmacher Institute, 2020. https://guttmacher.org/ survey-reproductive-health (accessed 17 April 2021).

6. Pattinson R, Fawcus S, Gebhardt S, Niit R, Soma-Pillay P, Moodley J. The effect of the first wave of COVID-19 on use of maternal and reprodetive health services and matent deats maternal and reproductive health services and maternal deaths https.//jourals.co.za/doi/ (accessed 17 April 2021) manuscript. item/9789240013131 (accessed 16 April 2021). early-impacts-covid-19pandemic-findings-2020-guttmacher-
7. Ahmed T, Rahman AE, Amole TG, et al. The effect of COVID-19 on maternal newborn and child health (MNCH) services in Bangladesh, Nigeria and South Africa: Call for a contextualised pandemic response in LMICs. Int J Equity Health 2021;20:77. pandemic response in LMICs. Int J Equity
https://doi.org/10.1186/s12939-021-01414-5

8. Adamu AA, Jalo RI, Habonimana D, Wiysonge CS. COVID-19 and routine childhood immunization in Africa: Leveraging systems thinking and implementation science to improve immunization system performance. Int J Infect Dis 2020;98:161165. https://doi.org/10.1016/j.ijid.2020.06.072

9. Kruger EC, Banderker R, Erasmus RT, Zemlin AE. The impact of COVID-19 on routine patient care from a laboratory perspective. S Afr Med J 2020;110(12):1201-1205. https://doi. org/10.7196/SAMJ.2020.v110i12.15294

10. Moultrie H, Kachingwe E, Ismail F. Impact of COVID-19 and Moultrie H, Kachingwe E, Ismail I. Impact of COVID-19 and public health restrictions on $\mathrm{TB}$ testing and case detection in South Africa. National Institute for Communicable Diseases, COVID-19 Special Public Health Surveillance Bulletin, 16 September 2020, vol. 18, supplementary issue 4. https://www.researchgate.net/publication/346396453_ Impact_of_Lockdown_on_TB_testing_in_South_Africa/ link/5fbf9e65458515b797703blb/download (accessed 10 May 2021).

11. Ahmed SAKS, Ajisola M, Azeem K; on behalf of the Improving Health in Slums Collaborative. Impact of the societal response to COVID-19 on access to healthcare for non-COVID-19 health issues in slum communities of Bangladesh, Kenya, Nigeria and
Pakistan: Results of pre-COVID and COVID-19 lockdown Pakistan: Results of pre-COVID and COVID-19 lockdown
stakeholder engagements. BMI Glob Health 2020;5:e003042. https://doi.org/10.1136/bmjgh-2020-003042

12. United Nations Population Fund (UNFPA). Impact of COVID-19 on family planning: What we know one year into the pandemic. Technical note, 11 March 2021. https://www. unfpa.org/sites/default/files/resource-pdf/COVID_Impact_FP_ V5.pdf (accessed 9 April 2021).

13. Kotlar B, Gerson E, Petrillo S, et al. The impact of the COVID-19 pandemic on maternal and perinatal health: A scoping review. Reprod Health 2021;18:10. https://doi.org/10,1186/s12978-02101070-6

14. United Nations Population Fund (UNFPA). COVID-19 technical brief package for maternity services. July 2020. https:// technical brief package for maternity services. July 2020. hitps://
www.unfpa.org/sites/default/files/resource-pdf/COVID-19 Www.unfpa.org/sites/default/files/resource-pdf/COVID-19Maternity_Services_TB_Pac
pdf (accessed 9 April 2021).

pdf (accessed 9 April 2021).
15. Kim AW, Nyengerai T, Mendenhall E. Evaluating the mental health impacts of COVID-19 pandemic in urban South Africa: Perceived risk of COVID-19 infection and childhood trauma predict adult depressive symptoms. medRxiv 2020 (epub 16 June 2020). https://doi.org/10:1101/2020.06.13.20130120

16. Pillay Y, Johnson L. World AIDS Day 2020: Reflections on global and South African progress and continuing challenges. S Afr J HIV Med 2021;22(1): a1205. https://doi.org/10.4102/sajhivmed. v22i1.1205

17. Pillay Y, Mvusi L, Mametja LD, Dlamini S. What did we learn from South Africa's first-ever tuberculosis prevalence survey?
frillay Y, Mvusi L, Mametja LD, Dlamini S. What did we learn S Afr Med J 2021;111(5):402-404. https://doi.org/10.7196/ SAMJ.2021.v111i5.15662

18. The Global Fund. The impact of COVID-19 on HIV, TB and malaria services and systems for health: A snapshot from 502 health facilities across Africa and Asia. April 2021. https://www. theglobalfund.org/media/10776/covid-19_2020-disruptionimpact_report_en.pdf (accessed 20 April 2021).

19. World Health Organization. Pulse survey on continuity of essential health services during the COVID-19 pandemic. 27 August 2020. https://www.who.int/publications/ $/$ /item/WHO2019-nCoV-EHS_continuity-survey-2020.1 (accessed 23 April 2019-1).

20. Wandai M. Aagaard-Hansen J, Sartorius B, Hofman K. Available data sources for monitoring non-communicable diseases and their risk factors in South Africa. S Afr Med J 2017;107(4):331337. https://doi.org/10.7196/SAMJ.2017.v107i4.11438

Accepted 4 May 2021. 